\title{
Imaging and Electron Energy-Loss Spectroscopy with Single Nanosecond Electron Pulses
}

\author{
Matthieu Picher ${ }^{1}$, Kerstin Bücker ${ }^{1}$, Thomas LaGrange ${ }^{2}$ and Florian Banhart ${ }^{1}$ \\ 1. Institut de Physique et Chimie des Matériaux, University of Strasbourg, CNRS, Strasbourg, France \\ 2. Interdisciplinary Center for Electron Microscopy, Ecole Polytechnique Fédérale de Lausanne, \\ Lausanne, Switzerland
}

Many dynamic processes in nanosystems happen at timescales beyond the shortest useful exposure times of TEMs with continuous beams. A higher temporal resolution has been achieved in the past years by applying pulsed electron beams in ultrafast and dynamic TEMs (UTEM/DTEM) [1, 2]. In a pumpprobe approach, where a transformation of the object is induced by a laser pulse, ultrashort electron pulses are used as probes for imaging, electron diffraction or EELS. Reversible transformations of the object can be studied with a stroboscopic technique where many pump-probe cycles with few-electron pulses are summed up to obtain data with sufficient signal/noise ratio. The stroboscopic technique is well-established in several laboratories and has produced outstanding results [1]. However, most lasertriggered structural transformations in materials are irreversible and can only be studied with one single pump-probe sequence [2]. Due to the necessity of using ultrashort pulses with $10^{7}-10^{9}$ electrons, electron-electron interaction within the pulses becomes important and produces restrictions on the spatial and temporal coherence as well as on the temporal and energy width of the pulses.

Here, we show the development of a TEM that is optimized for the stroboscopic as well as for the single-shot mode. The setup is based on a Jeol 2100 with thermionic emitter that has been upgraded for UTEM by IDES Inc. [3]. While the same femtosecond laser is used for pump and probe pulses in the stroboscopic mode, a nanosecond UV laser is necessary in the single-shot mode to generate intense photoelectron pulses with sufficient length (typically meters along the propagation direction) to reduce electron-electron interaction. The limiting factors in the single-shot mode are space charge around the cathode and the Boersch effect in the gun and lens cross-overs. This leads to decreasing coherence and increasing energy and temporal spread. A systematic study was undertaken to determine suitable settings for imaging and EELS in the single-shot mode [4]. The most important parameters are the UV laser intensity on the photocathode, the Wehnelt bias, and the gap between Wehnelt and the Ta disc cathode.

While single-shot imaging and diffraction studies have already been carried out earlier [2], single-shot EELS is shown in this study for the first time. Electron pulses with up to $10^{9}$ electrons propagating unobstructed from the cathode to the specimen (i.e., at low Wehnelt bias) show an energy width of hundreds of $\mathrm{eV}$ which is unsuitable for EELS. However, at high Wehnelt bias, the chromatic aberration of the Wehnelt produces an energy-dispersive plane in the source image so that the appropriate selection of the emission area can be used for energy filtering of the pulses. This considerably reduces the energy width, however at the expense of signal. With weak pulses, energy widths (FWHM of the zero-loss peak) of $2.1 \mathrm{eV}$ can be reached. Spectra with sufficient counting statistics, so that the core-loss peaks appear above the background, are obtained at energy widths of 5-10 eV.

Due to the large energy spread of the nanosecond electron pulses, the chromatic aberration of the objective lens has an influence on the resolution limit in imaging. This can be seen when the energy width is adjusted by changing the Wehnelt bias. Furthermore, the necessity to collect as many electrons 
as possible for an acceptable signal/noise ratio requires the focusing of a convergent beam onto the specimen and does not permit imaging with parallel illumination. This implies the influence of spherical aberration of the objective even at nanometer resolution. The aberration limits could be overcome in future developments by using aberration-corrected TEMs. Finally, shot noise is another limiting factor in imaging and EELS. The combination of spherical and chromatic aberration with shot noise limit the image resolution to tens of nanometers in the single-shot mode. Aberration correction and electron detection with higher sensitivity could improve the image resolution by an order of magnitude [5].

\section{References:}

[1] A. Zewail, Science 328 (2010) 187.

[2] W. E. King et al, J. Appl. Phys, 97 (2005), 111101.

[3] K. Bücker et al., Ultramicroscopy 171 (2016) 8.

[4] M. Picher, K. Bücker, T. LaGrange and F. Banhart, submitted.

[5] Funding by the EQUIPEX program of the Agence Nationale de Recherche (France), contract ANR11-EQPX-0041 (project UTEM) is gratefully acknowledged. The authors are grateful to B. Reed, S. T. Park and D. Masiel from IDES Inc. for helpful exchange of knowledge.
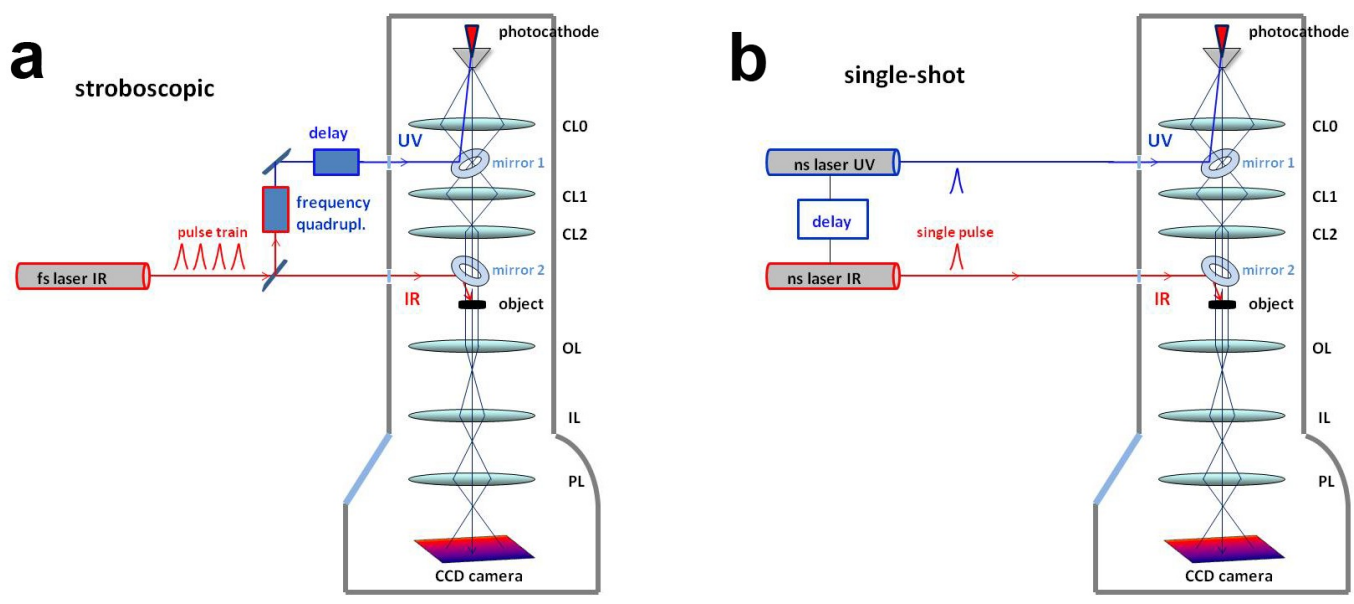

Figure 1. Schematics of the setup in the stroboscopic (a) and single-shot operation mode (b).
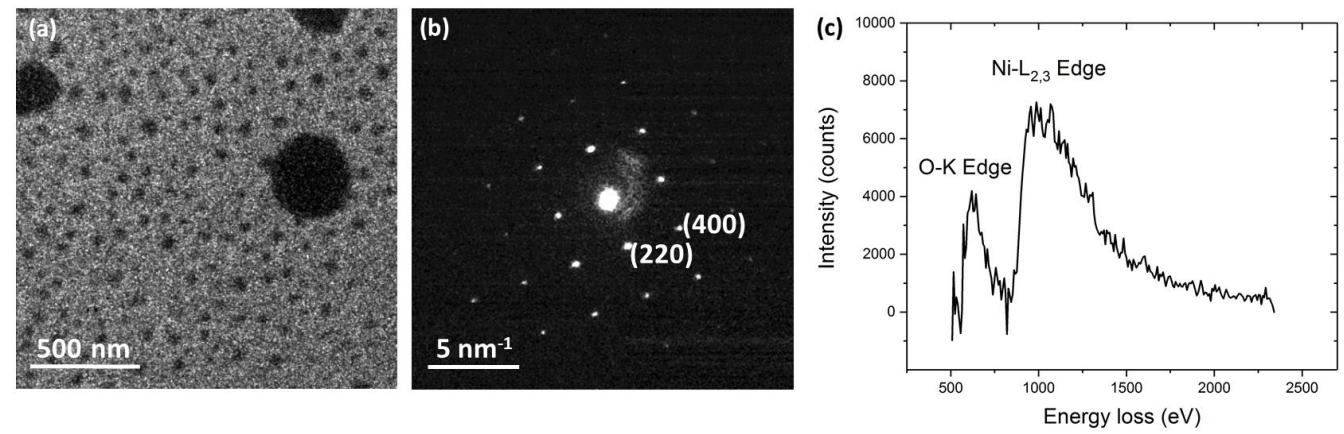

Figure 2. (a) Single-shot image (raw, no noise filtering) of gold particles on an amorphous carbon membrane. (b) Single-shot diffraction pattern of a Si monocrystal. (c) Single-shot EEL spectrum (background subtracted, no averaging) of a nickel oxide layer on a carbon membrane. Pulse length in (ac): $7 \mathrm{~ns}$. 